\title{
Local Adaptive Laplacian for Better 2-D Up-sampling
}

\author{
Aditya Acharya \\ Dept. of Electronics and Communication Engg. \\ National Institute of Technology Rourkela \\ Rourkela-769008, India \\ aditya.acharya2011@gmail.com
}

\author{
Sukadev Meher \\ Dept. of Electronics and Communication Engg. \\ National Institute of Technology Rourkela \\ Rourkela-769008, India \\ sukadevmeher@gmail.com
}

\begin{abstract}
Most of the existing up-sampling techniques through interpolation produce undesirable blurring artefacts while converting a low resolution image to its high resolution counterpart. The blurring is caused by the loss of high frequency information during the down sampling and upsampling process. To overcome this problem, a no reference, local adaptive, 2-D Laplacian based post processing technique is proposed here. The proposed method sharpens the DCT (Discrete cosine transform) based up-sampled video intraframe based on its local statistics so as to compensate the high frequency loss. Generally, the degree of blurring is more in the high variance regions in an image. Therefore, to restore the high frequency information, the sharpening should be more in the high variance regions than the low variance regions. Therefore, in this proposed method, a 2-D Laplacian kernel is made adaptive as per the statistical local variance of a $3 \times 3$ neighbourhood. If the local variance is more, the central kernel weight becomes proportionately high and vice versa based on the direct mapping basis. The remaining pixel weights of the Laplacian kernel are adjusted as per the central pixel weight such that the sum of all weights in the adaptive Laplacian kernel is zero. Experimental results reveal that the proposed method outperforms most of the widely used existing interpolation techniques in terms of objective and subjective measures.
\end{abstract}

Keywords-image and video processing; Laplacian; upsampling; interpolation; Discrete cosine transform; local variance

\section{INTRODUCTION}

Up-sampling plays a major role in image communication in restoring the high resolution 2-D signals from its low resolution counterpart at the receiver. Generally, at the transmitting end, a video intra-frame is sub-sampled to lessen the bandwidth required for transmission. At the receiver, the resolution of the sub sampled intra-frame is improved to the original by a suitable interpolation technique. This process not only lessens the signal bandwidth for transmission but also avoids channel congestion through a communication link. In addition, interpolation plays a significant role in various applications such as medical diagnosis, satellite image monitoring, video surveillance and many more. In such applications, it is very often required to improve the native resolution of the original image for proper inspection and recognition. For such operations, interpolation is used as a post processing step so as to improve the native resolution of the captured image for subsequent analysis and interpretation.

There are several interpolation techniques used for the up-sampling process. One of the simplest interpolation technique is bilinear interpolation where the value of a new point is computed using linear interpolation of four pixels surrounding the new point [1]. Bilinear interpolation though is simple and less complex, it has undesirable blurring artefacts. There are widely used interpolation techniques [2, 5] such as Bicubic and B-spline which consider sixteen pixels for determining a new interpolated point. These techniques provide better performance in terms of quality at the cost of computational complexities. Bicubic and B-spline interpolation techniques provide a less degree of blurring in comparison to bilinear interpolation. Lanczos is another spatial domain interpolation technique which is implemented by multiplying a sinc function with a sinc window which is scaled to be wider and truncated to zero outside of a range [6]. Even if Lanczos interpolation gives good results, it is slower than other approaches and provides a blurring effect in the reconstructed image. Many approaches for image resizing have been developed in transform domain [7, 8]. Up-sampling in DCT domain is implemented by padding zero coefficients to the high frequency side. Image resizing in DCT domain shows very good result in terms of scalability and image quality. However, this technique suffers through undesirable blurring and ringing artefacts.

Thus, there is a requirement of an efficient interpolation technique which not only gives a very less amount of blurring but also improves the subjective and objective quality of an up-sampled video intra-frame. The proposed local adaptive Laplacian based post processing technique sharpens the intra frame based on local statistics such that the high variance regions with more high frequency information are sharpened more than the low variance regions. Therefore, the proposed local adaptive Laplacian is a post processing technique which is based on an inverse modeling approach to counter the blurring problem and to preserve the fine details and edge information in an up-sampled intra-frame under various constraints.

The organization of the paper is structured as follows. The proposed method is described in the subsequent section. Section-3 provides the simulation results of different interpolation algorithms subjected to various constraints. Finally, the work is concluded in section- 4 . 


\section{PROPOSED METHOD}

In the proposed method, a sub-sampled video intra-frame is produced by alternate deletion of rows and columns at the transmitter for effective use of transmission channel bandwidth where as at the receiver, the resolution of the subsampled video is enhanced to its original size by DCT interpolation technique. In the final step, the DCT based upsampled intra-frame is sharpened adaptively as per the local statistics of a 3x3 neighbourhood by the local adaptive Laplacian kernel. This post processing technique is intended for alleviating the blurring which may arise due to DCT based up-sampling scheme. The proposed scheme is an inverse modeling approach of high frequency degradation which is meant for the restoration of high frequency contents.

Up-sampling using interpolation is analogous to LPF (low pass filter) operation as it shows the similar effects as that of LPF. Therefore, to combat the blurring problems caused by different interpolation techniques, the proposed method makes use of a local adaptive 2-D Laplacian based post processing technique. The proposed scheme sharpens the high variance regions more than the low variance regions using local adaptive 2-D Laplacian kernel. In this case, the central weight of Laplacian kernel is updated as per statistical local variance of a $3 \times 3$ neighbourhood on direct mapping basis. The remaining weights of Laplacian kernel are adjusted as per the central weight. Since the high frequency regions such as fine details and edges are more deteriorated than the slowly varying smooth regions during the up-sampling operation, the sharpening is made more in the high frequency region than the low frequency regions. This is meant to nearly equalize the uneven degree of blurring at different regions. In this way, the proposed method is based on inverse modeling approach is so as to nearly equalize the extent of blurring at different regions by adaptive sharpening. In addition, the region adaptive Laplacian kernel improves the adaptability of the proposed technique under various constraints such as change in compression ratio, zooming conditions and video types. On this basis, the proposed no reference post processing technique not only enhances the subjective and objective quality of the up-sampled video intra-frame but also gives much pronounced edge with very less degree of blurring and fine detail preservation under a variety of circumstances. The proposed method consists of three basic steps. They are namely

- Generation of low resolution or sub-sampled video intra frame by alternate deletion of rows and columns at 4:1 and 16:1 compression ratio respectively.

- 2-D up-sampling of low resolution video intra frame using the DCT interpolation technique to its original size.

- Local adaptive Laplacian based post processing.

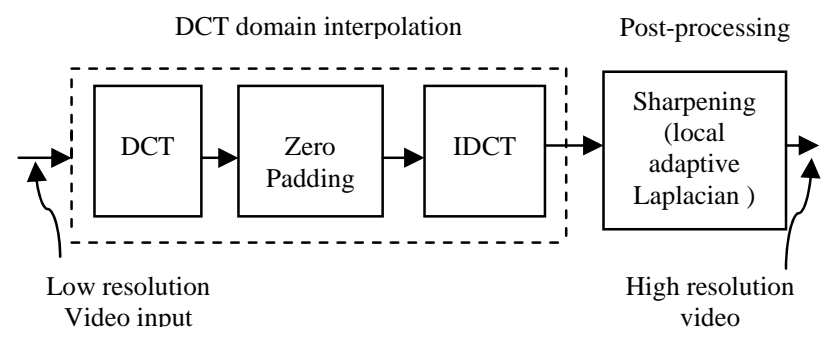

Figure 1. Local adaptive Laplacian based post processing

\section{A. Up-sampling in the DCT domain}

To implement up-sampling in DCT domain, we need to add $N$ zeros in the high frequency regions, where $N$ is the signal length. After that, type-II IDCT of the extended $2 N$ samples is performed to obtain the twofold up-sampled data. This process was described at length in [7]. In case of video frames, the up-sampling in a matrix form is given by

$$
b_{2 N \times 2 N}^{U}=W_{2 N \times 2 N}^{T} \times\left(\begin{array}{cc}
2 W_{N \times N} b_{N \times N} W_{N \times N}^{T} & 0 \\
0 & 0
\end{array}\right) \times W_{2 N \times 2 N}
$$

Where $W$ denotes the 1-D type-II DCT kernel. $b$ and $b^{U}$ are the down-sized and the up-sampled frame block. 0 is $N \times N$ zero matrix [8].

\section{B. Local adaptive Laplacian based post processing}

In this proposed method, initially the maximum local variance is calculated within an intra-frame. The local variance is then estimated for each pixel in a $3 \times 3$ neighbourhood within the intra frame. The estimated local variance is then normalized to 0 to 10 scale by dividing it with the maximum local variance and then by multiplying it with a scaling factor 10 . The remaining weights of the adaptive kernel are adjusted according to the central kernel weight such that the sum of all the weights of the adaptive Laplacian kernel is zero as shown in the equation (2). The adaptive Laplacian kernel is indicated as $h$ in the algorithm.

$$
\sum_{i=1}^{9} W t_{i}=0
$$

If the statistical local variance of a neighbourhood is more, so does the central weight of the 2-D Laplacian kernel on the direct mapping basis and vice versa. In this way, the weights of the local adaptive Laplacian kernel are updated by local variance so as to perform an adaptive sharpening based on local statistics. This provides the basis for the inverse operation of high frequency degradation and is meant for the restoration of high frequency details. Now the weighted output of the region adaptive Laplacian is added to the blurred up-sampled intra frame to generate the restored, deblurred video intra frame. The value of the weight factor $K$ differs for different compression ratio. At 4:1 compression ratio, the weight factor is kept 0.5 whereas at 16:1 
compression ratio the value is kept at 2.5. This is because, at lower compression ratio, the blurring is less and therefore the value of $K$ is kept low in order to perform adequately less amount of sharpening to equalize the less degree of blurring. On the other hand, at 16:1 compression ratio, the degree of blurring is more. Therefore, to lessen the high level of blurring, the value of $K$ is kept high. As a consequence, the regions with more textural and edge information are sharpened more than the flat or slowly varying regions in order to provide a better video quality.

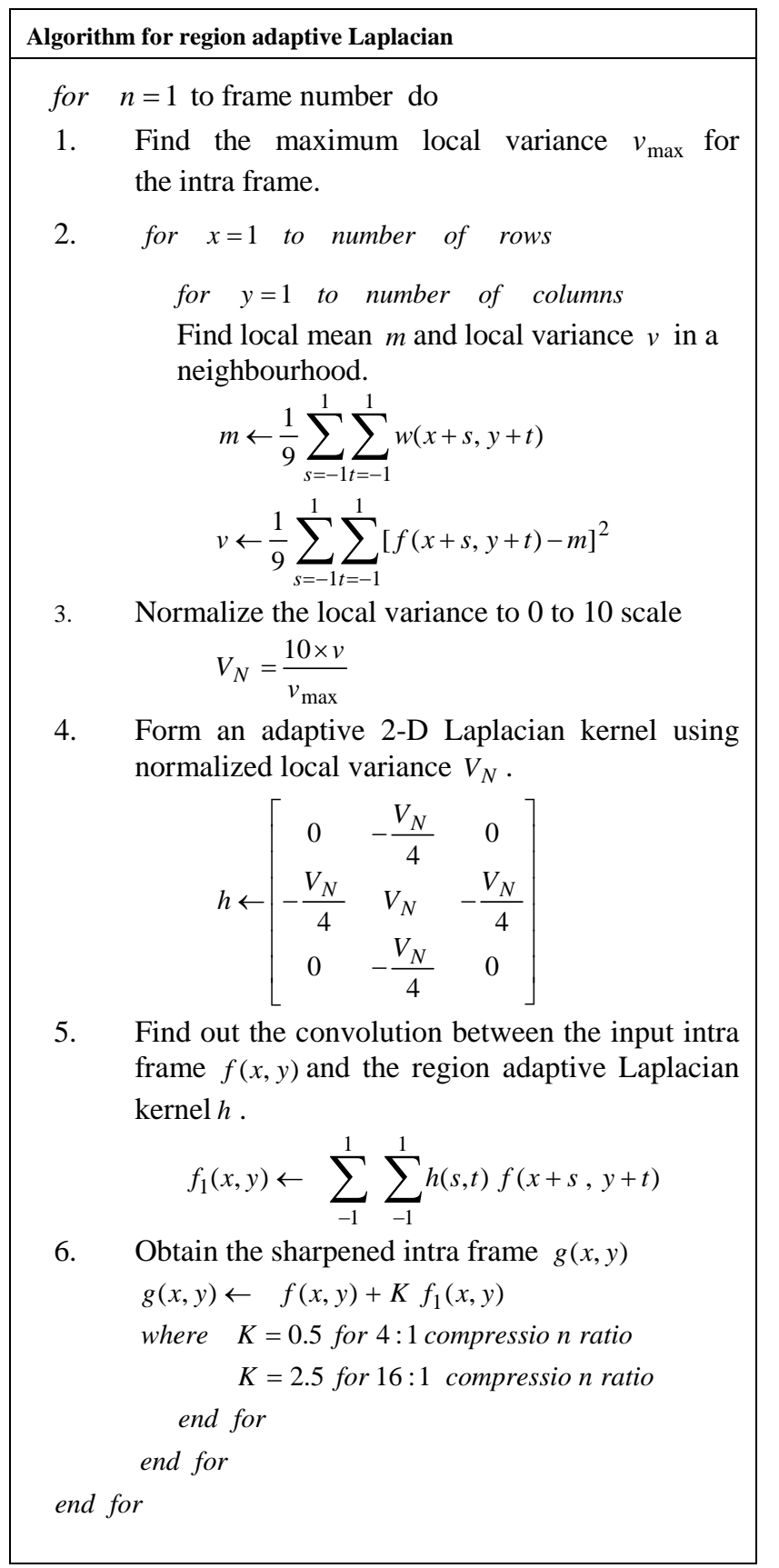

\section{EXPERIMENTAL RESULTS AND DISCUSSION}

To demonstrate the performance of the proposed postprocessing scheme, the input video sequences are downsampled in the spatial domain by deleting alternate rows and columns at $(4: 1)$ and $(16: 1)$ compression ratio respectively. Then for each scheme, we interpolate the frames back to their original size to allow the comparison with the original video. Table 1 and Table 2 illustrate the average PSNR comparison of DCT, Bicubic, Lanczos-3 and the proposed post-processing techniques at $4: 1$ and 16:1 compression ratios respectively. Experimental results reveal, at $4: 1$ compression ratio the proposed technique shows the average PSNR improvement up to $1.324 \mathrm{~dB}$ than DCT and an improvement up to $1.918 \mathrm{~dB}$ than the popular Bicubic interpolation particularly for ice sequence. At 16:1 compression ratio, the gain is up to $0.913 \mathrm{~dB}$ than DCT.

TABLE I. AVERAge PSNR COMPARISON OF DifFERENT SEQUENCES AT 4:1 COMPRESSION RATIO

\begin{tabular}{|c|l|l|l|l|}
\hline \multirow{2}{*}{$\begin{array}{c}\text { Video } \\
\text { sequences }\end{array}$} & \multicolumn{4}{|c|}{ Average PSNR (dB) } \\
\cline { 2 - 5 } & Bicubic & \multicolumn{1}{|c|}{ Lanczos-3 } & DCT & Proposed \\
\hline Ice & 80.7227 & 81.2724 & 81.3500 & $\mathbf{8 2 . 6 7 3 7}$ \\
\hline Football & 76.6988 & 77.4973 & 77.8178 & $\mathbf{7 8 . 4 9 2 3}$ \\
\hline Xylophone & 78.5179 & 79.0084 & 79.1473 & $\mathbf{8 0 . 0 3 5 9}$ \\
\hline Akiyo & 81.0421 & 81.5810 & 81.7782 & $\mathbf{8 2 . 5 7 3 6}$ \\
\hline City & 75.7227 & 76.0100 & 75.9829 & $\mathbf{7 6 . 2 1 3 8}$ \\
\hline Container & 73.6984 & 74.1394 & 74.3871 & $\mathbf{7 4 . 8 0 5 9}$ \\
\hline Mobile & 69.3281 & 69.7275 & 69.8889 & $\mathbf{7 0 . 3 7 6 5}$ \\
\hline Soccer & 78.3849 & 78.7956 & 78.8791 & $\mathbf{7 9 . 2 1 6 0}$ \\
\hline Stefan & 70.8642 & 71.1441 & 71.1566 & $\mathbf{7 1 . 3 8 8 5}$ \\
\hline Coastguard & 74.6310 & 75.0699 & 75.2115 & $\mathbf{7 5 . 4 8 1 0}$ \\
\hline
\end{tabular}

TABLE II. AVERAGE PSNR COMPARISON OF DIFFERENT SEQUENCES AT 16:1 COMPRESSION RATIO

\begin{tabular}{|c|l|l|l|l|}
\hline \multirow{2}{*}{$\begin{array}{c}\text { Video } \\
\text { sequences }\end{array}$} & \multicolumn{4}{|c|}{ Average PSNR (dB) } \\
\cline { 2 - 5 } & Bicubic & \multicolumn{1}{|c|}{ Lanczos-3 } & DCT & Proposed \\
\hline Ice & 75.5415 & 75.8449 & 75.9274 & $\mathbf{7 6 . 8 4 0 5}$ \\
\hline Football & 71.3216 & 71.5169 & 71.6358 & $\mathbf{7 1 . 9 4 0 4}$ \\
\hline Xylophone & 73.6599 & 73.8457 & 73.9412 & $\mathbf{7 4 . 3 7 3 8}$ \\
\hline Akiyo & 76.2144 & 76.4112 & 76.4478 & $\mathbf{7 6 . 8 9 2 1}$ \\
\hline City & 72.3711 & 72.4679 & 72.4509 & $\mathbf{7 2 . 5 4 9 8}$ \\
\hline Container & 69.6644 & 69.7546 & 69.7354 & $\mathbf{6 9 . 8 0 2 5}$ \\
\hline Mobile & 65.8343 & 65.9303 & 65.9213 & $\mathbf{6 6 . 0 7 1 1}$ \\
\hline Soccer & 74.2612 & 74.4484 & 74.4829 & $\mathbf{7 4 . 7 2 5 0}$ \\
\hline Stefan & 67.6652 & 67.7742 & 67.8474 & $\mathbf{6 7 . 9 7 0 5}$ \\
\hline Coastguard & 70.6563 & 70.7569 & 70.7682 & $\mathbf{7 0 . 8 8 5 8}$ \\
\hline
\end{tabular}




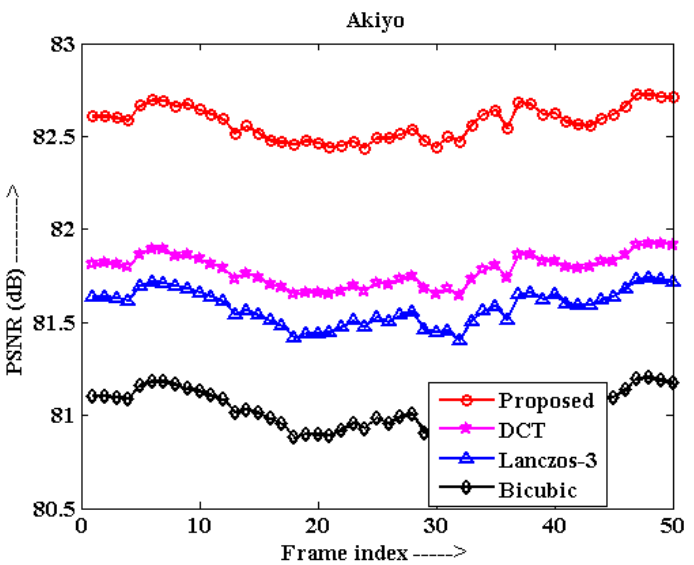

(a)

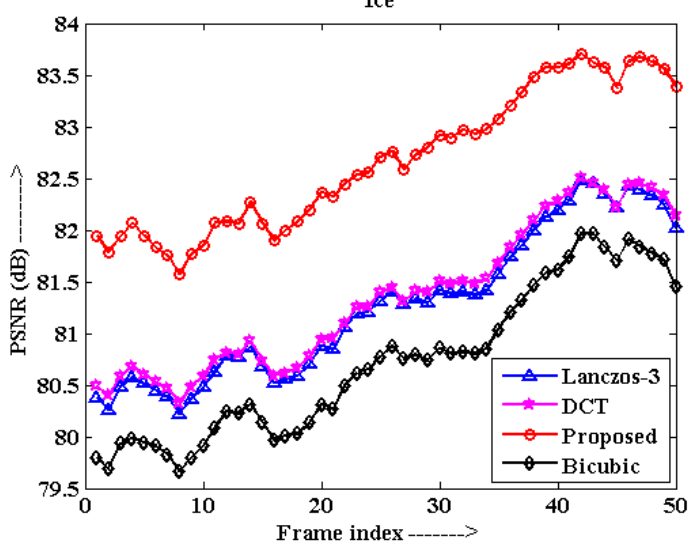

(b)

Figure 2. PSNR comparison at 4:1 compression ratio: (a) akiyo; (b) ice .

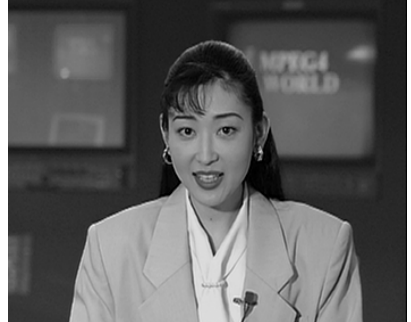

(a)

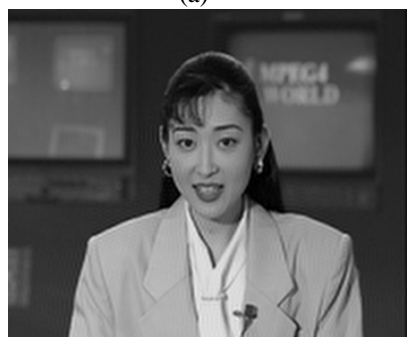

(c)

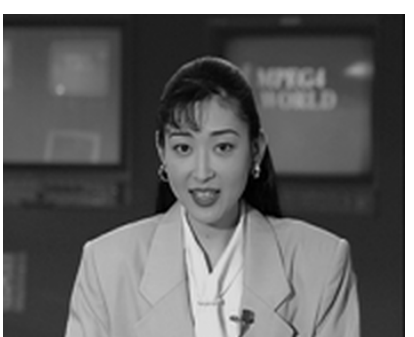

(b)

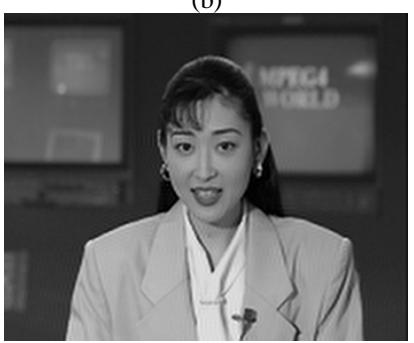

(d)
Figure 3. Subjective performance of $33^{\text {rd }}$ frame of akiyo sequence at $4: 1$ compression ratio: (a) Original; (b) Lanczos-3; (c) DCT; (d) Proposed.
In Figure 2 the variations of PSNR w.r.t the frame index are shown at 4:1 compression ratio. In this case, the proposed method yields better PSNR gain than the other widely used interpolation techniques for different types of sequences. The subjective performance of the proposed technique is illustrated in Figure 3 for the 33rd frame of akiyo sequence at $4: 1$ compression ratio.

\section{CONCLUSION}

The proposed post-processing scheme is based on an inverse modeling approach for high frequency degradation which restores the fine details and edge information of a video i.e. lost during the downsampling and up-sampling process. In addition, this alleviates the problem of nonlinear blurring caused by such operation. The improvement is gained by using the local statistics based adaptive Laplacian kernel whose weights are updated as per the statistical local variance of a neighbourhood on direct mapping basis. This post-processing scheme not only restores a sub-sampled video with high precision but also yields a very low degree of blurring with fine detail preservation. In addition, the proposed scheme performs quite adaptively under various constraints such as change in compression ratio, zooming conditions and the video types and provides much better performance. As per experimental evidences, the proposed scheme shows significant PSNR gain of $1.324 \mathrm{~dB}$ than DCT at 4:1 compression ratio. Moreover, The subjective quality improvement of the proposed technique can be notified in terms of much pronounced edge, less degree of blurring and fine details preservation under a variety of circumstances.

\section{REFERENCES}

[1] Lu Jing, Xiong Si, Wu Shihong, “An improved bilinear interpolation algorithm of converting standard defination images to high defination images,” WASE Int. Conf. on Info. Engg. pp.441-444, 2009.

[2] R. G. Keys, "Cubic convolution interpolation for digital image processing,” IEEE Trans. Acoust., speech, signal Process., vol. ASSP29, no.6, pp.1153-1160, Dec.1981.

[3] S. E. Reichenbach and F.Geng, "Two-dimensional cubic convolution,” IEEE Trans. Image Process., vol.12, no.8, pp.857-865, Aug. 2003.

[4] Zhou Dengwen, "An edge directed bicubic interpolation algorithm,” CISP, pp.1186-1189, 2010.

[5] H. S. Hou and H. C. Andrews, "Cubic splines for image interpolation amd digital filtering” IEEE Trans. Acoust., speech and sign. Proc., vol. ASSP-26, 1978.

[6] Wenxing Ye, Alireza Entezari, A geometric construction of multivariate sinc functions, IEEE Transaction on Image processing 2011; 19(12).

[7] R. Dugad and N. Ahuja, "A fast scheme for image size change in the compressed domain,” IEEE Trans. Circuit, Syst., Video Technology., vol. 11, pp. 461-474, Apr, 2001.

[8] Zhenyu Wu, Hongyang Yu, and Chang Wen Chen, "A new hybrid DCT-Wiener based interpolation scheme for video intraframe upsampling," IEEE signal processing letters, vol. 17. No. 10, pp. 827830, oct. 2010. 